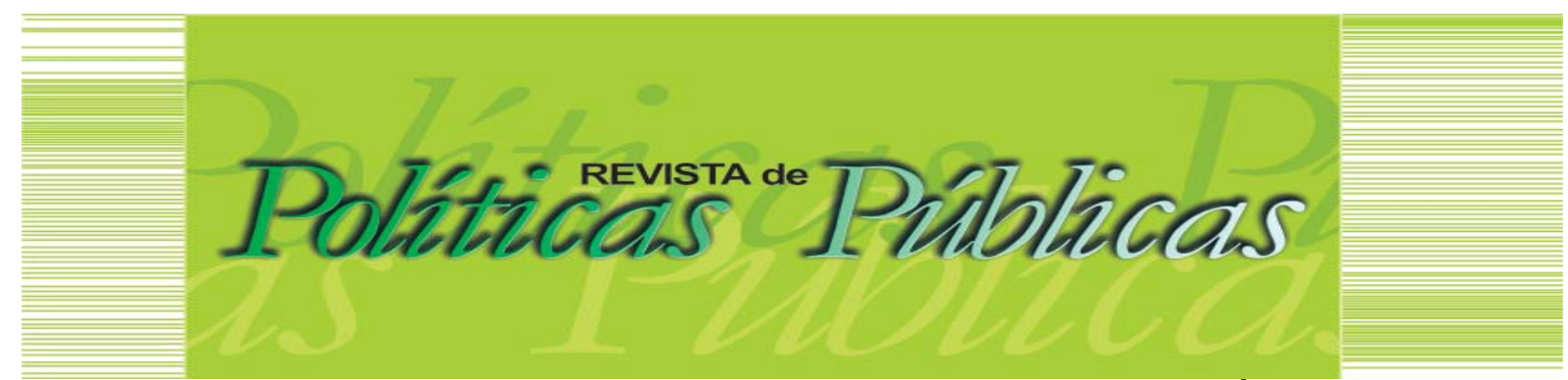

\title{
IMPACTOS DEL COVID-19 E INSTRUMENTOS DE APOYO A LA PRODUCCIÓN EN MUNICIPIOS METROPOLITANOS ARGENTINOS
}

Rodrigo Reynaldo Carmona ${ }^{1}$

\section{Resumen}

El artículo busca examinar los impactos recientes del COVID-19 sobre el entramado productivo argentino y los principales instrumentos de apoyo desarrollados, en particular en relación a los municipios del Área Metropolitana de Buenos Aires. Primeramente, plantea los debates vinculados a la problemática productiva en el espacio metropolitano, el despliegue de políticas asociadas y las perspectivas frente al COVID-19. Seguidamente, analiza los efectos de la pandemia sobre la producción manufacturera y el empleo, los principales instrumentos de apoyo desarrollados a nivel nacional y provincial y las acciones desplegadas desde los municipios hacia las firmas. Por último, hace unas reflexiones finales sobre los impactos del COVID-19 en relación a la producción industrial, el alcance de los instrumentos generados y los principales desafíos que aparecen en los municipios seleccionados.

Palabras claves: Impactos COVID-19, instrumentos de apoyo, producción, municipios

\section{IMPACTS OF COVID-19 AND INSTRUMENTS TO SUPPORT PRODUCTION IN ARGENTIINE METROPOLITAN MUNICIPALITIES}

\begin{abstract}
The article seeks to examine the recent impacts of COVID-19 on the Argentine productive network and the main support instruments developed, in particular in relation to the municipalities of the Buenos Aires Metropolitan Area. First, the debates related to the productive problem in the metropolitan area, the deployment of associated policies and the perspectives against COVID-19 are raised. Next, the effects of the pandemic on manufacturing production and employment, the main support instruments developed at the national and provincial level, and the actions deployed from the municipalities to the firms are analyzed. Finally, some final reflections are made on the impacts of COVID-19 in relation to industrial production, the scope of the instruments generated and the main challenges that appear in the selected municipalities.

Keywords: COVID-19 impacts. Support instruments. Production. Municipalities
\end{abstract}

Artigo recebido em: 22/04/2021 Aprovadoem: 30/11/2021

DOI: http://dx.doi.org/10.18764/2178-2865.v25n2p 890-907

\footnotetext{
1 Doctor en Ciencias Sociales por la Facultad Latinoamericana de Ciencias Sociales, Mención Honorífica (FLACSO-Sede Argentina). Profesor e investigador ordinario del área "Estado, Gobierno y Administración Pública" del Instituto del Conurbano (ICO) dela Universidad Nacional de General Sarmiento (UNGS) e investigador de carrera adjunto del Consejo Nacional de Investigaciones Científicas y Técnicas (CONICET). Coordinador de Investigación ICO-UNGS y es Director conjunto de la Diplomatura en Administración y Gestión de Organizaciones Públicas. E-mail: rcarmona@campus.ungs.edu.ar
} 


\section{INTRODUCCIÓN}

El presente trabajo1busca examinar los impactos recientes del COVID-19 sobre el entramado productivo argentino y los principales instrumentos de apoyo desarrollados, en particular en los municipios del Área Metropolitana de Buenos Aires (AMBA). El análisis contempla desde la instrumentación del Aislamiento Social, Preventivo y Obligatorio (ASPO) a comienzos de la pandemia, hasta las nuevas medidas de Distanciamiento Social, Preventivo y Obligatorio (DISPO)2.El territorio a considerar comprende los 24 municipios/partidos del Gran Buenos Aires, que rodean a la Ciudad Autónoma de Buenos Aires, y junto con esta última aportan casi la mitad de la riqueza del país.

La actividad industrial tiene un peso significativo para la población que vive en los municipios dentro del AMBA, puesto que en la producción manufacturera se genera un enorme conjunto de puestos de trabajo, muchos de ellos con salarios relativamente altos y buenas condiciones laborales. En la industria del Gran Buenos Aires, además, se procesan diversas materias primas, partes e insumos producidos en otras zonas del país y se generan productos exportables y divisas siendo esta actividad un gran dinamizador de la economía. Estos aspectos justifican la importancia de examinar lo que está sucediendo en este segmento productivo ante la irrupción de la pandemia por COVID-19y los distintos instrumentos de apoyo desplegados en el área metropolitana más grande del país.

El trabajo se organiza del siguiente modo. En primer lugar, se considera el marco conceptual en relación a la dinámica productiva en el marco de ciudades metropolitanas, el despliegue de políticas públicas y las perspectivas ante el COVID-19. En segundo término, se hace una caracterización de la evolución de la actividad industrial y la situación a comienzos del año 2020, y luego se contemplan los resultados de diversas entrevistas realizadas a diversos referentes públicos y privados en relación a: los impactos del ASPO y DISPO en la producción manufacturera y el empleo, los principales instrumentos de apoyo desarrollados desde el Estado nacional y provincial, las acciones desplegadas desde el nivel local hacia las firmas respecto a las restricciones productivas y la implementación de protocolos por la emergencia sanitaria, acciones de reconversión e innovación frente a la pandemia y la utilización de alternativas tecnológicas como el teletrabajo. Por último, se hacen unas reflexiones finales sobre los impactos del COVID-19 en la producción industrial, el alcance de los instrumentos de apoyo y las perspectivas que se abren en los municipios considerados. 


\section{DINÁMICA PRODUCTIVA METROPOLITANA, POLÍTICAS Y PERSPECTIVAS ANTE EL COVID-19}

La estructura y dinámica económica del Gran Buenos Aires en el marco más amplio del $\mathrm{AMBA}^{3}$, está fuertemente condicionado por su histórica posición de centralidad en la vida económica argentina (Gráfico 1). Esta área concentra la mayor parte de la actividad industrial y financiera del país, además de agrupar casi de un tercio de la población total (13 millones de habitantes). Este proceso de concentración, se explica, según BORELLO, FRITZSCHE y VÍO (2000), por los factores de atracción propios de las ciudades metropolitanas, como los servicios a la producción, la especialización del mercado de trabajo y, fundamentalmente en esta última década, el acceso a la información.

Figura 1 - El Área Metropolitana de Buenos Aires

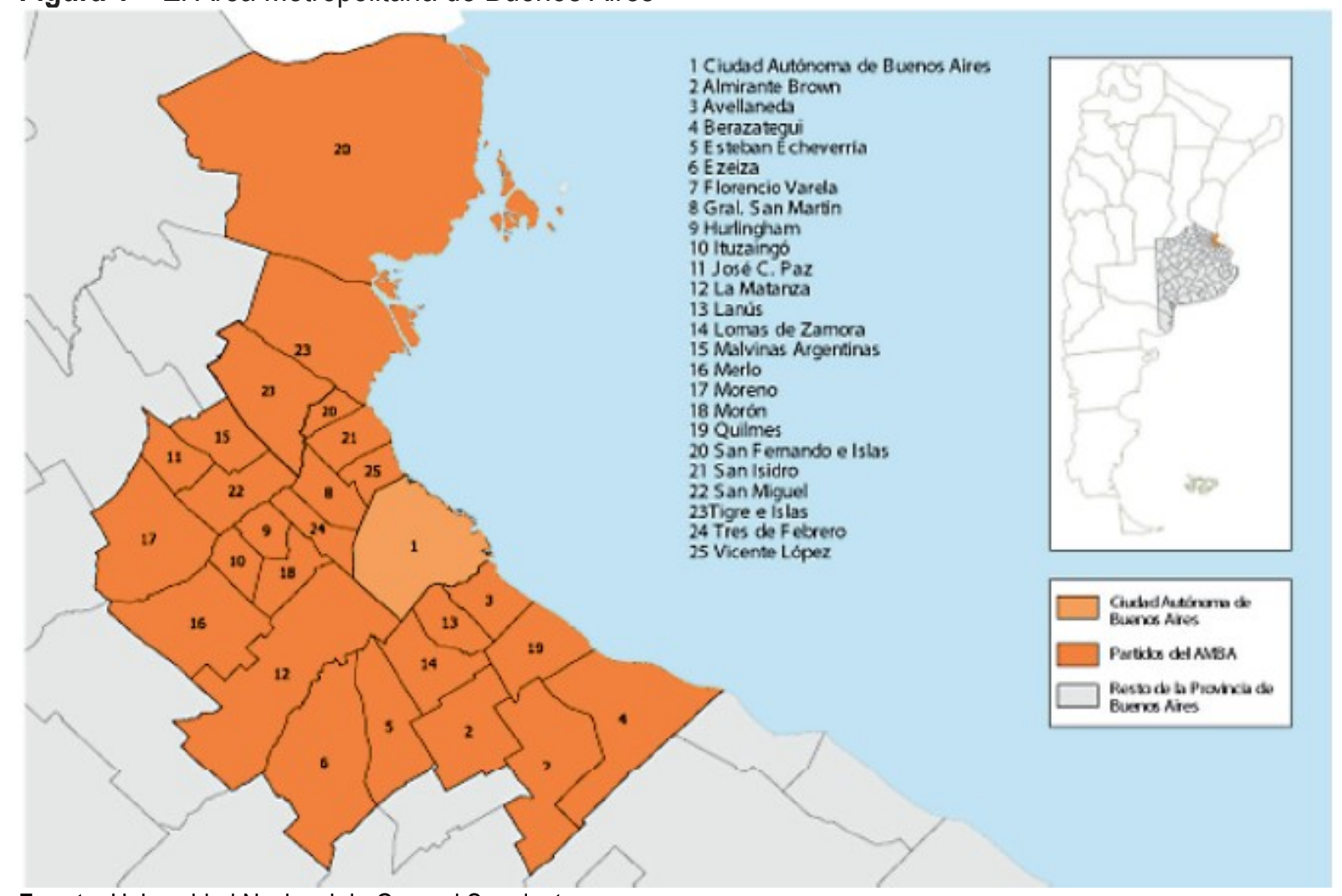

Fuente: Universidad Nacional de General Sarmiento

Estas tendencias se expresan con nitidez en la situación relativa del mercado de trabajo respecto del ámbito nacional. Este territorio muestra, según el Instituto Nacional de Estadística y Censos (INDEC), la tasa de actividad más elevada del país a la vez que enfrenta situaciones de desempleo y subempleo más críticas que otras áreas metropolitanas. La estructura productiva mantiene así los rasgos fundamentales de su configuración tradicional, y no se ha alterado sustancialmente la tendencia histórica de localización industrial, consistente en un proceso de periferización, desde el centro de la ciudad de Buenos Aires hacia los partidos del conurbano más 
alejados (BORELLO; FRITZSCHE; VÍO, 2000; FRITZSCHE; VÍO, 2002, BORELLO, 2007; FAL; CARMONA, 2016).

Como resultado de estos procesos, la estructura económica y productiva del AMBA muestra un alto grado de heterogeneidad, configurándose una estructura territorial fragmentada entre algunos centros o ejes dinámicos y grandes espacios de estancamiento o crecimiento limitado. Esta estructura es producto de factores que operan principalmente a escala metropolitana, puesto que las decisiones de inversión se toman en función del mercado de tierras, de servicios y de mano de obra de la metrópoli en su conjunto (BORELLO, 2007; BORELLO; YOGUEL, 2000; CARMONA; COUTO, 2019 y 2020).

De esta manera lo metropolitano constituye una unidad amplia y diversa de procesos y estructuras urbanas, que constituye el resultado del crecimiento de una ciudad, y por lo tanto, supera los límites del gobierno municipal que fue creado para hacerse cargo de su gestión. Los procesos económicos, sociales, políticos y ambientales que se desarrollan en una región metropolitana se basan en lógicas de acción que responden a distintas escalas. Por un lado, existe la escala metropolitana que abarca a los procesos que tienen como referencia el conjunto territorial. En segundo lugar, este espacio participa en circuitos más amplios, de carácter nacional, supranacional y global. Por último, es posible también identificar escalas submetropolitanas: nos referimos con ello a los niveles subregional y local, asumiendo que este último concepto puede hacer referencia, en el caso de ciudades grandes, al espacio del municipio, al de la localidad o, también, al del barrio (BORELLO et al., 2004; CARMONA, 2009).

Estas escalas se encuentran determinadas por los ámbitos de referencia territorial de los distintos procesos y cuestiones que constituyen la dinámica de crecimiento de la ciudad metropolitana, es decir, aspectos físico materiales, sociales, económicos, político institucionales y culturales. Ello supone que será posible encontrar problemáticas que se despliegan completamente en una escala y otras que incluyan más de una o que atraviesen esta delimitación. Así, los procesos que sustentan la dinámica de las regiones metropolitanas se rigen por lógicas territoriales de distinto nivel.

Desde una dimensión económica, si bien la escala metropolitana es la que rige para los circuitos de producción y circulación de mayor importancia económica, paralelamente coexisten mercados sub-metropolitanos, donde circulan funciones y actividades de menor relevancia pero que posiblemente asuman un papel más importante en la reproducción cotidiana de los hogares populares. Es decir, si bien la localización de las actividades económicas más concentradas se distribuye en función de criterios propios de una ciudad metropolitana, en esta aglomeración conviven circuitos económicos de menor escala, determinados por la proximidad geográfica, tanto en el mercado de 
trabajo, de tierra y vivienda, como en la circulación y consumo de productos. (BORELLO, 2007; FAL; CARMONA, 2016).

En estos términos, la complejidad espacial metropolitana es el resultado de la coexistencia de una diversidad de ámbitos territoriales diferentes, que se constituyen con relación a los procesos, cuestiones y actores que organizan la vida de la ciudad. Desde una perspectiva económica y geográfica, si bien el área metropolitana es una unidad, es posible en términos de flujos identificar subunidades más acotadas que pueden ser desagregadas en términos de municipio, localidad, barrio 0 corredor productivo y de transporte.

En este marco, la irrupción de la pandemia constituye lo que las ciencias sociales denominan un "hecho social total" en tanto impacta sobre el conjunto de las relaciones sociales, territorios y estremece a la totalidad de los actores, instituciones y valores. Con la pandemia, la incertidumbre como hecho abarcador se hace evidente y muchos de los déficits de las sociedades y metrópolis se agudizan, lo cual llevan a la necesidad de repensar las estructuras de gestión y gobernanza de modo de poder responder más efectivamente a fenómenos y emergencias cada vez más complejas (CARMONA; COUTO, 2020; PONT-VIDAL, 2020).

Las administraciones públicas deben, por lo tanto, replantear sus modalidades de actuación de forma de avanzar en nuevos esquemas de intervención que ganen en flexibilidad, adaptación y legitimidad en un contexto incierto y cambiante. Esto resalta la necesidad de coordinar esfuerzos institucionales y sociales en esta coyuntura excepcional, de modo de plasmar formas de gobernanza multinivel y territorial de nuevo tipo (CARMONA, 2012, 2015; VIGIL; FERNÁNDEZ, 2012; SUBIRATS, 2015).

En efecto, tal como destaca GARCÍA DELGADO (2020) las distintas dimensiones de la relación Estado-sociedad han quedado afectadas por el COVID-19 y los efectos de la crisis económica mundial: la de acumulación; la de legitimación; de inserción internacional; la cultural, así como la de articulación con la sociedad civil. En un escenario caracterizado por la incertidumbre y la complejidad que con la pandemia, según PONT VIDAL (2020) se vuelve hipercompleidad, las ciudades y metrópolis aparecen en una situación ambivalente. Por un lado, ganan protagonismo al organizar y dar cuenta de la atención sanitaria, de los grupos vulnerables y proponer políticas para la reapertura de la economía y el comercio local. Por otro lado, los Estados-Nación y las regiones recuperan preponderancia global, mientras que el marco normativo y de intervención local sigue siendo precario. Ello determina la importancia de generar relaciones intergubernamentales más consistentes, a la vez que nuevas formas de intervención pública más coordinadas, integrales y en articulación con los actores sociales. 


\section{ESTRATEGIA METODOLÓGICA}

El trabajo parte de un abordaje metodológico mayormente cualitativo, con la combinación de datos de tipo cuantitativo. En primer término, con el objeto de hacer una caracterización de la evolución de la actividad industrial y la situación a comienzos del año 2020 se examinan diversas estadísticas e informes sobre la producción manufacturera.

En segundo lugar, se consideran los resultados de diversas entrevistas realizadas a referentes púbicos y privados vinculados con la actividad productiva en municipios del Gran Buenos Aires. Los principales ejes contemplados son los siguientes: impactos del ASPO y DISPO en la producción y el empleo, los principales instrumentos de apoyo desarrollados desde el Estado nacional y provincial, las acciones desplegadas desde el nivel local hacia las firmas respecto a las restricciones productivas y la implementación de protocolos por la emergencia sanitaria, acciones de reconversión e innovación frente a la pandemia y la utilización de alternativas tecnológicas como el teletrabajo.

Este trabajo se circunscribe territorialmente a los 24 partidos/municipios que conforman el denominado Gran Buenos Aires en el AMBA y las actividades examinadas son las ramas de actividad que implican transformación o ensamble y que son incluidas en la industria manufacturera de acuerdo al Instituto Nacional de Estadísticas y Censos (INDEC). En términos institucionales y a nivel de actores productivos, se contemplan respuestas de 35 entrevistados: secretarios de producción y de industria de los gobiernos municipales, referentes de cámaras empresarias, sindicatos y gremios, gerentes de parques industriales y propietarios de firmas industriales.

\section{PRODUCCIÓN INDUSTRIAL Y COVID-19 EN MUNICIPIOS DEL AMBA}

\subsection{Evolución y escenario pre-pandemia}

Distintos indicadores generales de la actividad industrial exhiben en los últimos años, una tendencia a la baja en los volúmenes de producción en el AMBA. Más allá de las variaciones estacionales y mensuales en los volúmenes de producción, por ejemplo, la serie del Índice de Producción Industrial Manufacturero (IPI manufacturero)4muestra una clara tendencia a la baja entre los años 2016 y 2019 para el conjunto de la actividad industrial del país (Figura 2). 
Figura 2 - PI manufacturero nivel general. Serie original, desestacionalizada y tendencia-ciclo, base 2004=100, en números índice. Enero 2016-marzo 2020

Indice

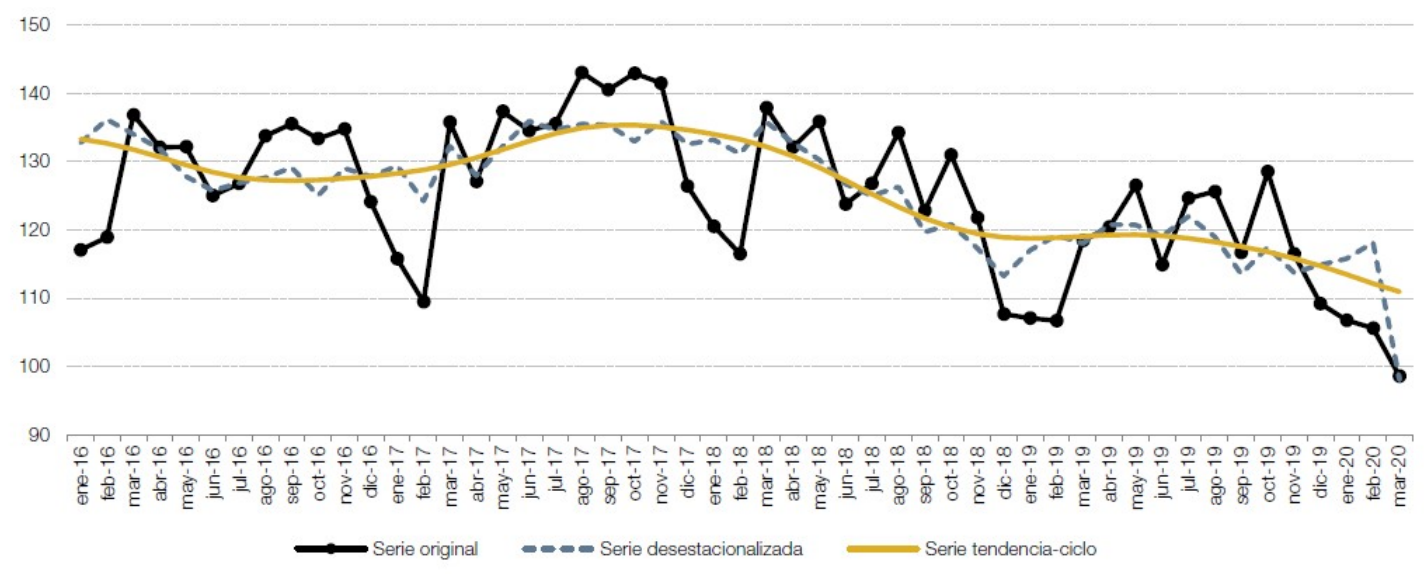

Fuente: INDEC 2020

Si bien estos números se refieren al país en su conjunto, por el propio peso del Gran Buenos Aires en el total nacional, bien podemos suponer que también se ha dado una tendencia al estancamiento o a la caída en su producción industrial. En este sentido, el $50 \%$ del producto bruto geográfico industrial se concentra en cinco municipios, General San Martín, Vicente López, La Matanza, Avellaneda y Tres de Febrero ubicados mayormente en el primer cordón del área metropolitana.

Del mismo modo, el conjunto de los partidos del Gran Buenos Airesconcentra cerca del $27 \%$ del empleo registrado de la industria manufacturera de todo el país, siendo una de las zonas más importantes en términos de la participación del empleo industrial dentro del total del empleo formal del sector privado. Sin embargo, atraviesa un proceso de declive paulatino -en relación al total del empleo registrado en el plano territorial y a nivel agregado en todo el país. La tabla 1 muestra la evolución de la participación del empleo industrial tanto en el plano de estos partidos como respecto al ámbito nacional. Puede verse así como en los últimos 25 años, la industria en esos partidos perdió cerca de 12 puntos porcentuales de participación respecto al total del empleo privado registrado, mientras que a nivel nacional la caída fue de unos 8 puntos.

Tabla 1 - importancia relativa del empleo industrial del gran buenos aires en el total del empleo registrado, 1996-2019

\begin{tabular}{|l|c|c|c|c|c|c|}
\hline Porcentajes & 1996 & 2001 & 2004 & 2011 & 2015 & 2019 \\
\hline $\begin{array}{l}\text { GBA Industria } \\
\text { empleo total } \\
\text { registrado }\end{array}$ & $38,9 \%$ & $30,7 \%$ & $31,8 \%$ & $30,3 \%$ & $29,3 \%$ & $26,9 \%$ \\
\hline
\end{tabular}




\begin{tabular}{|l|c|c|c|c|c|c|}
\hline \multicolumn{2}{|l|}{ GBA } & \multicolumn{2}{|l|}{} & & \\
\hline $\begin{array}{l}\text { GBA Industria } \\
\text { nacional } \\
\text { empleo } \\
\text { registrado en } \\
\text { la industria }\end{array}$ & $25,7 \%$ & $21,0 \%$ & $21,4 \%$ & $20,1 \%$ & $19,7 \%$ & $17,9 \%$ \\
\hline
\end{tabular}

Fuente: Observatorio de Empleo y Dinámica Empresarial - Ministerio de Trabajo, Empleo y Seguridad Social.

En efecto, en términos absolutos, con años recesivos entre 2011 y 2019 se perdieron cerca de 110 mil puestos de trabajo registrados en el sector industrial, de los cuales más de 35 mil correspondieron a los partidos del Gran Buenos Aires. En su conjunto, se pasa de registrar 363.219 empleos industriales en 2014 a contabilizar 328.324 empleos el año pasado según la tabla 1 en base los datos del Observatorio de Empleo y Dinámica Empresarial - Ministerio de Trabajo, Empleo y Seguridad Social.

Para el primer semestre de 2020, los datos provisorios de la misma fuente arrojan casi 322 mil empleos industriales. En la tabla 2 se presenta la evolución del empleo manufacturero entre 2014 y 2020, por grupos de ramas industriales.

Tabla 2 - Evolución del empleo por rama (valores absolutos)

\begin{tabular}{|l|c|c|c|c|c|c|c|}
\hline Ramas & 2014 & 2015 & 2016 & 2017 & 2018 & 2019 & $2020^{*}$ \\
\hline $\begin{array}{l}\text { Industria } \\
\text { alimenticia }\end{array}$ & 63.938 & 65.104 & 65.500 & 64.740 & 63.833 & 61.669 & 60.660 \\
\hline $\begin{array}{l}\text { Industria } \\
\text { automotriz }\end{array}$ & 33.334 & 31.918 & 31.166 & 30.467 & 29.633 & 25.851 & 25.441 \\
\hline $\begin{array}{l}\text { Industrias } \\
\text { de proceso }\end{array}$ & 61.547 & 64.081 & 65.005 & 63.800 & 62.684 & 60.492 & 61.255 \\
\hline $\begin{array}{l}\text { Intensivas } \\
\text { en } \\
\text { ingeniería }\end{array}$ & 37.241 & 38.137 & 37.772 & 36.815 & 36.140 & 34.478 & 33.502 \\
\hline $\begin{array}{l}\text { Intensivas } \\
\text { en mano } \\
\text { de obra }\end{array}$ & 145.547 & 151.423 & 151.023 & 145.479 & 137.768 & 126.595 & 122.634 \\
\hline $\begin{array}{l}\text { Intensivas } \\
\text { en recursos } \\
\text { naturales }\end{array}$ & 21.613 & 22.333 & 21.899 & 21.501 & 20.891 & 19.550 & 18.488 \\
\hline \begin{tabular}{l} 
Total \\
\hline
\end{tabular} & 363.219 & 372.996 & 372.364 & 362.802 & 350.949 & 328.634 & 321.981 \\
\hline
\end{tabular}




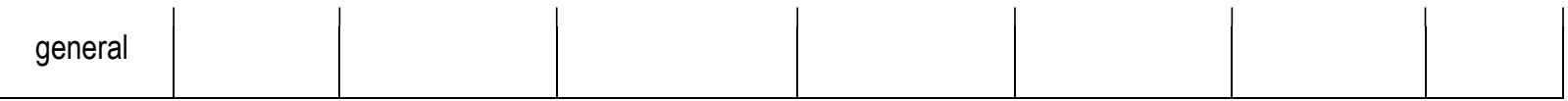

Fuente: Observatorio de Empleo y Dinámica Empresarial - Ministerio de Trabajo, Empleo y Seguridad Social.

En promedio hay una caída del $10 \%$ del empleo para el total de las ramas industriales, aunque hay varios grupos de actividades que registran una caída significativamente mayor, como es el caso de la industria automotriz y, en menor medida, las ramas intensivas en mano de obra. Todas las otras ramas registran guarismos que están cerca del promedio para el Gran Buenos Aires o en un nivel mucho más favorable. Pero el impacto absoluto es algo diferente ya que algunos conjuntos de ramas tienen un gran peso en el empleo total, como es el caso de las ramas intensivas en mano de obra. De hecho, ese conjunto de ramas explica la caída de dos tercios de la pérdida total de empleos industriales registrados en ese período.

\subsection{Arribo de la pandemia, impactos en la producción y respuestas gubernamentales e institucionales}

La producción industrial en el Gran Buenos Airesse desarrolla en un escenario dificultoso, de fuerte impacto inicial de la enfermedaden el conjunto territorial aunque en caída de contagios en los últimos meses. La producción industrial de estos partidos se plasma en los primeros meses en un contexto de restricciones a la movilidad y al despliegue de diversas actividades. En ese marco de situación, el gobierno nacional, pero también el provincial y losgobiernos municipales, dispusieron diversas acciones y mecanismos para afrontar el funcionamiento de la actividad económica en general y de la actividad industrial en particular. Este acompañamiento y apoyo se combinó también con el accionar de otras instituciones, como ser las cámaras empresarias ylos sindicatos,comoveremos más adelante.

Según información oficial sobre la evolución de la actividad industrial estimada del conjunto del país para el período enero-agosto de este año yen función de la fuerte participación en ese conjunto que alcanza al Gran Buenos Aires, en la figura 3 puede apreciarse la enorme caída de la actividad industrial a partir de mediados de marzo. Si bien ya a comienzos de mayo se reabre una serie de actividades industriales, inicialmente los niveles de actividad se mantuvieron bajos, aunque crecientes. Para fines de julio y comienzos de agosto y septiembre, los niveles de actividad empezaban a acercarse a los niveles pre-pandemia. Es más, hay algunos indicadores de actividad que son relativamente alentadores (por ejemplo, una mayor utilización de capacidad instalada), claro que los índices son calculados contra niveles muy bajos de actividad y de empleo, que son los registrados el año pasado. 
Figura 3 - Evolución de la actividad industrial estimada en Argentina. Enero-agosto, 2020

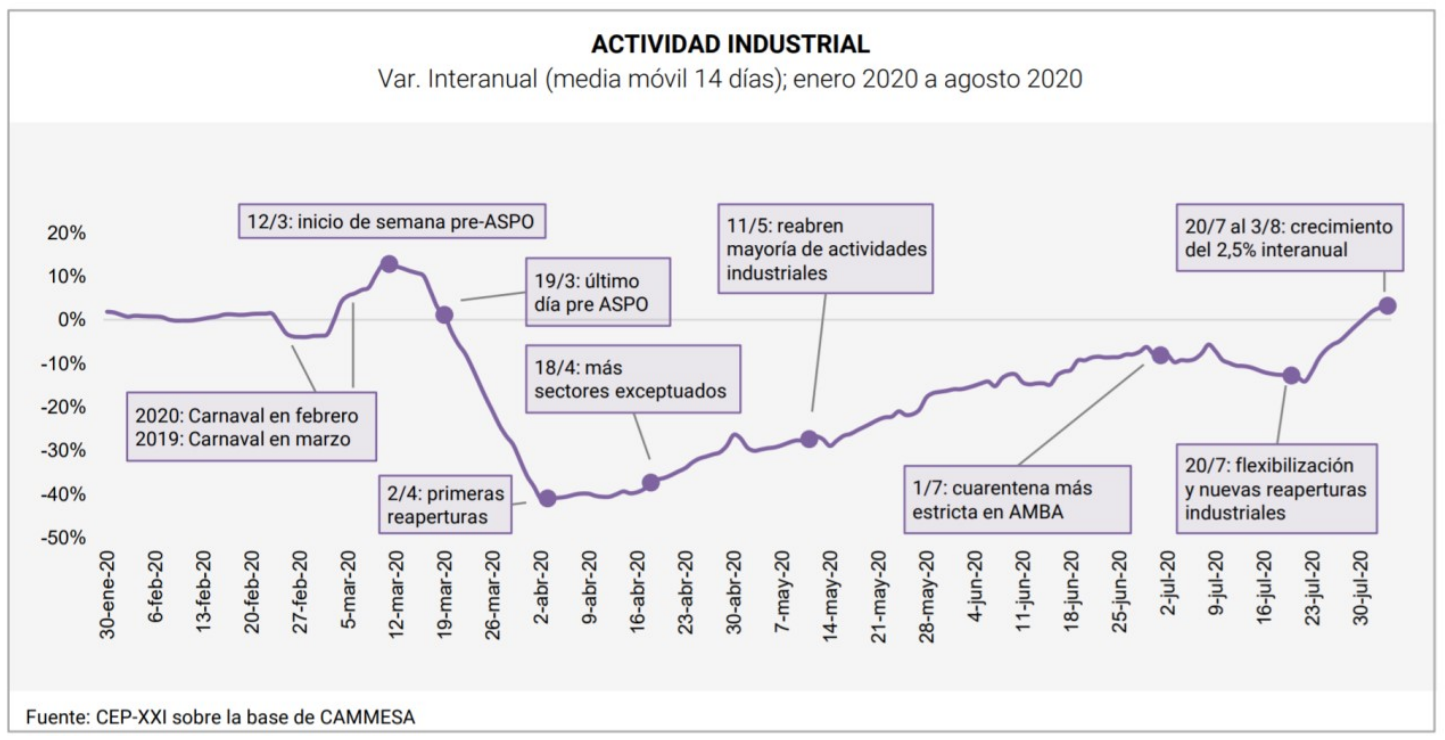

Fuente: CEP-XXI, 2020 "Panorama productivo".

Evolución de los principales indicadores de la actividad productiva", agosto 2020.

Es posible identificar dos conjuntos de acciones estatales. Por un lado, aparecen las medidas asociadas al proceso de ASPO, que son medidas destinadas a restringir la circulación y el contacto entre personas con el objetivo de limitar los contagios. Por otro lado, hay una serie de acciones destinadas a resolver un conjunto de problemas generados en las actividades económicas y en la población en general por el desarrollo de la propia enfermedad. En ese sentido, el Estado desplegó toda una batería de acciones que involucraron a diversos ministerios y agencias gubernamentales.Se pusieron en marcha así desde los inicios medidas tales como la prohibición de despidos, la suspensión de aumentos de tarifas y de cortes de servicios públicos, la ampliación del Programa de Recuperación Productiva (REPRO) -con el objeto de subsidiar una suma fija remunerativa de un monto equivalente al salario mínimo de empresas en crisis-, y el descuento y postergación de cargas patronales.

Estas acciones se encuadrarian posteriormente en el Programa de Asistencia de Emergencias al Trabajo y la Producción (ATP). En el marco de este programa el Estado nacional cubre parte del salario de los trabajadores privados en relación de dependencia en empresas afectadas por la caída de la actividad económica. También se incrementa el monto del seguro de desempleo, se otorgan créditos a tasa cero a autónomos y monotributistas, y se establecen a prórrogas y reducciones de hasta $95 \%$ de las contribuciones patronales. A ello se sumarían, también, créditos subsidiados a empresas PyMEs y la ampliación de la cobertura social y previsional, junto al Ingreso Familiar de Emergencia (IFE), mediante el pago de un bono a casi 9 millones de personas orientado a trabajadoras y trabajadores informales o desempleados. Posteriormente, a partir del DISPO con una apertura 
gradual de actividades se empezaron a desplegar nuevos instrumentos vinculados a comercio exterior y apoyo competitivo de las empresas.

Este conjunto de medidas representaría un $5 \%$ del PIB y un monto estimado respecto al gasto fiscal y las facilidades financieras de 1,4 billones de pesos. En la tabla 3se resumen los diferentes instrumentos otorgados por el Estado nacional y la Provincia de Buenos Aires en materia de asistencia a empresas.

Tabla 3 - Instrumentos de apoyo a las empresas en respuesta a la pandemia en Argentina

\begin{tabular}{|l|l|l|l|l|}
\hline \multicolumn{1}{|c|}{ Denominación } & \multicolumn{1}{|c|}{ Ámbito } & \multicolumn{1}{|c|}{ Tipo (crédito / subsidio) } & Tasa de interés & \multicolumn{1}{|c|}{ Organismo/s ejecutor/es } \\
\hline $\begin{array}{l}\text { Asistencia al Trabajo y la } \\
\text { Producción. Línea Salarios }\end{array}$ & Nacional & Créditos subsidiados & $0 \%$ & $\begin{array}{l}\text { Jefatura de Gabinete de } \\
\text { Ministros ANSES/ AFIP }\end{array}$ \\
\hline $\begin{array}{l}\text { Asistencia al Trabajo y la } \\
\text { Producción. Linea Créditos }\end{array}$ & Nacional & Créditos subsidiados & $0 \%$ & $\begin{array}{l}\text { Jefatura de Gabinete de } \\
\text { Ministros ANSES/ AFIP }\end{array}$ \\
\hline MiPyme & Nacional & Créditos subsidiados & $24 \%$ & $\begin{array}{l}\text { Banco Central de la República } \\
\text { Argentina }\end{array}$ \\
\hline PymePlus & Nacional & Créditos subsidiados & $24 \%$ & Ministerio de Producción \\
\hline $\begin{array}{l}\text { Preservar Trabajo (PPT) } \\
\text { Programa de Apoyo al sistema } \\
\text { productivo nacional }\end{array}$ & Provincial & Créditos subsidiados & $0 \%$ & $\begin{array}{l}\text { Ministerio de Trabajo de la } \\
\text { Provincia de Buenos Aires }\end{array}$ \\
\hline $\begin{array}{l}\text { Programa de Apoyo a la } \\
\text { Competitividad (PAC) y Aportes } \\
\text { No Reembolsables (ANR) para } \\
\text { empresas, instituciones de I+D+i, } \\
\text { universidades, } \\
\text { tecnológicos y emprendedores. }\end{array}$ & Nacional & Créditos subsidiados & $12 \%$ & $\begin{array}{l}\text { Ministerio de Desarrollo } \\
\text { Productivo }\end{array}$ \\
\hline
\end{tabular}

Fuente: Elaboración propia en base a información oficial relevada.

En el desarrollo de las distintas etapas de la pandemia, los gobiernos municipales y sus áreas de producción fueron instrumentando diversas acciones en materia de prevención, asistencia y apoyo a las empresas en cada uno de los distritos. A partir de entrevistas desarrolladas a funcionarios de municipios con importante actividad industrial (San Martín, Malvinas Argentinas, Quilmes, Tigre, Tres de Febrero, La Matanza), se planteó inicialmente la importancia de la instrumentación de los protocolos. En ese sentido, se destacó que luego de una primera fase de fuerte restricción de las actividades se fueron habilitando gradualmente la apertura de industrias. Se especificó que los pedidos elevados para estas aperturas comenzaron con un requerimiento a las empresas que solicitaron la exención de la cuarentena para que "con carácter de declaración jurada, garanticen el traslado de los trabajadores y las trabajadoras sin la utilización del transporte público de pasajeros y que adopten los protocolos sanitarios aprobados, mediante el Decreto 459/2020, para las actividades industriales respecto de las cuales solicita la excepción". 
El proceso continuaría con el aval de la Provincia y, finalmente, de la Jefatura de Gabinete nacional termina habilitando el regreso a la producción de las industrias en rubros tales como automotriz y autopartista; de indumentaria; metalúrgica, maquinaria y equipos; del calzado; gráfica, ediciones e impresiones; de la madera y muebles; juguetes; textil; química y petroquímica; y de plásticos y sus subproductos. No obstante, el inicio de esas actividades, conforme a lo que estipula el Decreto 340/2020 del Gobierno bonaerense, queda sujeto, en cada distrito, al dictado de un acto administrativo por parte de Ejecutivo local pertinente, "previa intervención y análisis de los gobiernos nacional y provincial".

Según lo referenciado por los funcionarios, luego de una primera etapa de la cuarentena con un funcionamiento sólo de un tercio de las empresas, vinculadas a actividades esenciales 0 ligadas, se pasó desde julio en adelante a la apertura de más de las dos terceras partes de las industrias (no de manera completa, con protocolos y en diferentes turnos) y en la actualidad el que pudo abrir sus puertas ya lo hizo. Además, de la fuerte caída de ventas en gran parte de las firmas al inicio -con excepción de los rubros esenciales-, se afirma que desde septiembre se observa un mayor nivel de actividad y utilización de la capacidad instalada.

Las acciones desplegadas, según el grado de consolidación institucional de las áreas de producción locales y las particularidades de la trama industrial de cada municipio, han sido múltiples. Además de la implementación de los protocolos y viabilizar los múltiples instrumentos crediticios y de asistencia productiva a nivel nacional y provincial, se han desarrollado iniciativas propias de capacitación laboral y digital (marketing digital, trabajo de plataformas), comercialización y armado de portales de empresas, programas de asistencia técnica general (principalmente a Pymes) y apoyo específicos para sectores afectados fuertemente por la pandemia (incluyendo en algunos casos además de empresas industriales, apuntalamiento al comercio y turismo). A ello se suman, acciones en términos de comercio exterior y apoyo a la competitividad -por ejemplo, en diseño e innovación, alimentos, transformación digital- en el marco de una mayor apertura de las actividades.

La articulación institucional desde los municipios ha sido amplia con distintas universidades (Universidad Nacional de San Martín, Universidad Nacional de Tres de Febrero, Universidad Nacional de Quilmes, Universidad Tecnológica Nacional, Universidad Nacional de General Sarmiento, Universidad Nacional de La Matanza), otros actores del sistema científico-tecnológico (Consejo Nacional de Investigaciones Científicas y Tecnológicas, Instituto Nacional de Tecnología Industrial, Ministerio de Ciencia y Tecnología), productivo (Secretaría PyME, Ministerio de Producción de Nación y Provincia de Buenos Aires, cámaras locales, sindicatos), ambiente (Ministerio de Medio Ambiente), cámaras de diverso grado, instancias cooperativas y bancarias (Fundación CREDICOOP, Santander Río, Banco Nación y Provincia), agrupamientos sectoriales (Fundación Pro Tejer, Asociación 
de Industriales Metalúrgicos de la República Argentina) y políticos (Produce Buenos Aires -con 50 Secretarios de Producción de la Provincia de Buenos Aires-, Consorcio de municipios del Oeste), con el objeto de dar respuesta tanto en términos sanitarios como presentar acciones de coordinación y colaboración más amplias en el apoyo a las empresas.

Si bien el impacto de la pandemia sobre el entramado productivo ha sido fuerte, algunos sectores han podido adaptarse mejor o reconvertirse, por ejemplo en rubros tales como el textil camisolines y barbijos-, plástico, química y caucho -separadores para hospitales y mamparas de acrílico protectoras, burletes, alfombras sanitizantes, paneles led, alcohol en gel y productos de limpieza- y bioseguridad -dispensadores de alcohol y botiquines, máscaras-. Del mismo modo, también se han desarrollado iniciativas de transformación digital 4.0 y diagnósticos de eficiencia energética. En algunos casos, como en San Martín, se han logrado desplegar acciones muy innovadoras, por ejemplo, un proyecto de alerta temprana utilizando inteligencia artificial para detectar el COVID y otros virus con apoyo de la Universidad Nacional de San Martín. En la misma línea, en Tres de Febrero se desarrollaron inversiones en materia de robótica y automatización (mayormente, en empresas vinculadas al sector alimenticio, consumo masivo y segundas/terceras marcas). También se observa, como en el caso de La Matanza, el impulso al desarrollo de un parque científico-tecnológico y a priorizar acciones estratégicas en términos de potenciar la economía del conocimiento, la cuestión medio ambiental y de género.

Se resalta en general que la situación productiva y laboral en los municipios es compleja y heterogénea, variando según el sector y también por tamaño de empresa. Se observa así una reactivación paulatina pero variable. De este modo, se pueden apreciar algunas situaciones exitosas, con casos de reconversión, incorporación de tecnología e inversiones en ciertos sectores, si bien predominan las firmas con dificultades y restricciones (en ciertos casos, con rebrotes de contagios ante la mayor actividad, aunque en caída desde mediados de octubre y noviembre). Los sindicatos así intervinieron fuertemente en la implementación de los protocolos sanitarios.

Muchas instituciones privadas han seguido con atención la evolución de la pandemia y las medidas de restricción, así como las respuestas de las empresas y de la demanda. En ese sentido, se han realizado relevamientos especiales para monitorear la evolución de la actividad y del empleo en las empresas asociadas. En primer lugar, señalan los mayores costos para las empresas que surgen de que muchas firmas han asumido los costos de transporte (el porcentaje en el Gran Buenos Aireses muy alto), de que muchas han hecho testes preventivos entre sus trabajadores y de que han tenido parte de su personal de licencia por razones de edad, enfermedad (COVID-19) o situaciones pre-existentes. En segundotérmino, destacan las dificultades de endeudamiento que enfrentan las empresas y el hecho 
que sólo parcialmente está pudiendo acceder las empresas a instrumentos específicos diseñados por el Estado, aunque las situaciones son muy dispares a nivel sectorial y regional.

Estas cuestiones son resaltadas,destacadas por entrevistas desarrolladas a cámaras locales, parques industriales y dirigentes sindicales. En términos de impacto a nivel del empleo, se señala mayormente que las empresas han ido acompañando los sueldos con fondos nacionales desde los primeros meses, con diversos establecimientos que han suspendido o desvinculado personal. Muchas firmas han buscado acumular stocks y ante la caída de ventas disminuir costos. Hubo mayormente reducción de horas extras, adelantos de vacaciones, y en algunos casos, para aprovechar al personal ocioso, se realizaron tareas de mantenimiento de las instalaciones. Por otro lado, la adopción de teletrabajo se ha extendido en las empresas con mayor tamaño y grado de consolidación, sobre todo en tareas administrativas, marketing, comercialización, mantenimiento de software y en menor medida en la producción.

De este modo, una porción importante de las empresas mantendrá su dotación de empleados al mismo nivel hasta que haya una reactivación mayor de la economía y que la situación de su negocio mejore. Por otra parte, aparece en el último tiempo una demanda de perfiles laborales específicos (como ser diseñadores, programadores y operadores de imprenta plástica) y las firmas más dinámicas en el actual escenario están teniendo dificultades para conseguir personal especializado.

En las empresas, la mayor parte (alrededor de un 80\%) ha solicitado el ATP y, en algunos casos, han solicitado créditos al $24 \%$ para pagar los sueldos. La mayoría de las empresas contactadas (el $70 \%$ no pertenece a una cámara empresaria. Este hecho es más que significativo, puesto que en este contexto de la pandemia no han tenido acceso aningún tipo de asesoramiento de estas organizaciones empresarias sobre protocolos a utilizar en la empresa para evitar riesgos de contagios. Este hecho ha llevado a los dueños y directivos de estas empresas a buscar otras fuentes de información sobre cómo proceder a través de contactos personales en los municipios, o con otros colegas, o a través de los sindicatos a los que pertenecen sus trabajadores y basado en el propio criterio de los empresarios.

Por último, gran parte de las empresas entrevistadas mencionan que necesitan de un crédito de un monto mayor para poder seguir manteniendo sus gastos o realizar algún tipo de inversión productiva. Por ello, es común que se endeuden con diferentes entidades. Cabe destacar que, de las 13 empresas entrevistadas, 10 solicitaron al menos un subsidio o crédito para afrontar la situación. 


\section{CONSIDERACIONES FINALES}

Un análisis general de la producción industrial en el Gran Buenos Aires muestra una situación bastante heterogénea, con actividades, procesos productivos, tamaños de empresas y localizaciones muy variadas. La pandemia aparece en un escenario con diversas limitaciones en ese entramado, algunas de larga data y otras de coyuntura. De este modo, la emergencia sanitariapone en evidencia problemas diversos en el aparato productivo e institucional. Por un lado, hay cuestiones históricas vinculadas con las limitadas capacidades de muchas firmaspara una buena implementación de protocolos, el acceso a programas de apoyo o la organización del teletrabajo. Por otro lado, muchas empresas que venían trabajando en condiciones recesivas y con bajo uso de su capacidad instalada han podido mejorar su performance.

La información analizada sugiere que se están alcanzando, en diversas empresas y sectores, niveles de uso de la capacidad instalada mayores que los que había antes de la pandemia. Ello, igualmente, no alcanza a todas las firmas y ramas. En un marco de reactivación gradual se pueden apreciar algunas situaciones exitosas, con casos de reconversión, incorporación de tecnología e inversiones (como por ejemplo, en el sector alimenticio, distribución o en relación a la atención sanitaria), si bien predominan las firmas con dificultades y restricciones.

En relación a la contención de la emergencia sanitaria, el Estado nacional ha desarrollado una amplia batería de instrumentos junto con la articulación con los nivelesprovincial y municipal. De la misma forma, diversas instituciones públicas y privadas de apoyo a la producción han colaborado y buscado resolver los problemas asociados a la pandemia. Se han destinado cuantiosos recursos a estos esfuerzos. Sin embargo, se aprecian limitaciones importantes en el propio funcionamiento del sistema productivo examinado, en el desarrollo de capacidades y el establecimiento de vínculos entre empresas entre sí y con las instituciones.

La crisis permitió observar también el surgimiento de iniciativas de fuerte valor desde lo innovador y desde el aprovechamiento de recursos con los que el país cuenta en su sistema empresarial, gubernamental y científico. La crisis no sólo desnuda las debilidades del aparato productivo sino que permite identificar recursos y capacidades que estaban subutilizados. Este factor positivo debería ser parte explícita de las acciones y políticas que se desarrollen en el momento de la post-pandemia.

El desarrollo de protocolos sanitarios y la apertura gradual de actividades estuvieron sujetos a las restricciones impuestas por la pandemia. Se plasmaron así espacios de coordinación e intercambio continuo entre las diferentes autoridades en el plano nacional, provincial y local, con un papel importante de los municipios en la aplicación e implementación de las medidas. 
En este contexto, se hace relevante seguir fortaleciendo las estructuras de gobernanza de modo de dar respuestas efectivas a situaciones cada vez más complejas. En función deello y particularmente en materia productiva para los municipios del AMBA examinados, resulta fundamental ver cómo generar mejores relaciones tanto a nivel intergubernamental como desde el conjunto de instituciones que operan en el territorio.

Finalmente, en términos de desafíos estratégicos para estimular dinámicas más virtuosas de desarrollo se destacan: i) la importancia de reconocer y rearmar cadenas de valor en los municipios, junto a tener mayor información disponible de las empresas; v) combinar la posibilidades de virtualidad y presencialidad; vi) elevar capacidades de las firmas, buscando la adopción de tecnologías de la industria 4.0 y mejorar la vinculación con cámaras y universidades. Estos factores resultan centrales en unescenario limitado por la crisis sanitaria y aún de transicióncon la llegada de las primeras vacunas, que requerirá de intervenciones claras y efectivas para apuntalar el entramado productivo local/regional y sostener los niveles de empleo.

\section{REFERÊNCIAS}

BORELLO, José (coord.) (2007).Aproximaciones al mundo productivo de la Región Metropolitana de Buenos Aires, Ediciones UNGS, Provincia de Buenos Aires, Argentina.

BORELLO, José; CARMONA, Rodrigo; CARIDE, Horacio; BETTATIS, Clarisa (2004). ¿Qué es lo local en laregión del Gran Buenos Aires? Elementos para definir unidades subregionales que seanútiles para encarar acciones de desarrollo local, con énfasis en el empleo. Informe final, presentado al Programa Área, Oficina Internacional del Trabajo (OIT), Oficina de Buenos Aires.

BORELLO, José; VÍO, Marcela; FRITZSCHE, Federico (2000). La geografía de la industria en la Región Metropolitana de Buenos Aires. Un análisis de los últimos censos económicos (19851994).Serie Informes de Investigación, n. 10, Instituto del Conurbano, UNGS, Provincia de Buenos Aires, Argentina.

BORELLO, José; YOGUEL Gabriel (2000). Algunas reflexiones sobre el ambiente local y las capacidades innovativas de las firmas, BORELLO. José (coord.), Bulones y Canguros, Los ejes productivos del desarrollo local, Instituto del Conurbano, UNGS, Provincia de Buenos Aires, Argentina.

CARMONA, Rodrigo; COUTO, Bárbara (2020a). Dinámica productiva, laboral e institucional en la esfera urbana. Un análisis crítico en municipios del Conurbano Bonaerense.Revista Quid 16, IIGG, Nro.13, Facultad de Ciencias Sociales, UBA, Ciudad Autónoma de Buenos Aires, Argentina. Disponible en: https://dialnet.unirioja.es/servlet/articulo?codigo $=7448700$

CARMONA, Rodrigo; COUTO, Bárbara (2020b). La gobernanza metropolitana frente a la crisis por la pandemia COVID-19. El caso del Área Metropolitana de Buenos Aires en Argentina. Deliberativa

Revista de Estudios Metropolitanos en Gobernanza, 2, e200204-NEM1.T1, Guadalajara, México. 
Disponible en:https://deliberativa.com/wp-content/uploads/2020/09/DREMeG-e200204-NEM1.T1Carmona-y-Couto.pdf

CARMONA, Rodrigo; COUTO, Bárbara. (2019). Políticas públicas e intervención institucional en materia de producción y empleo. Algunas consideraciones en municipios del noroeste del Conurbano Bonaerense. Revista Perspectivas de Políticas Públicas, Universidad Nacional de Lanús. Vol. 8 Núm. 16, Provincia de Buenos Aires, Argentina..Disponible en:

http://revistas.unla.edu.ar/perspectivas/article/view/2353

CARMONA, Rodrigo (2009). Sistemas productivos locales en el noroeste de la Región Metropolitana de Buenos Aires. Un análisis de su dinámica reciente y distintas consideraciones en términos de políticas públicas. RevistaEspaço de Diálogo e Desconexão - REDD-, Vol. 1 № 2 - 2009, San Pablo, Brasil.Disponible en: http://seer.fclar.unesp.br/index.php/redd/issue/view/367

CEP XXI (2020). Informe de panorama productivo. Evolución de los principales indicadores de la actividad productiva. Buenos Aires: CEP XXI- Centro de Estudios para la Producción/Ministerio de Desarrollo Productivo, agosto, Ciudad Autónoma de Buenos Aires. Disponible en:https://www.argentina.gob.ar/sites/default/files/ok_informe_de_panorama_productivo__diciembre_2020_version_resumida_0.pdf

FAL Juan; CARMONA, Rodrigo (2016). Instituciones, desarrollo local y políticas para la generación y fortalecimiento del empleo. Un análisis en municipios de la Zona Noroeste de la Región Metropolitana de Buenos Aires. Ponencia presentada en XXI Reunión Anual de la Red PyMEsMercosur. Provincia de Buenos Aires, Argentina.

FRITZSCHE, Federico; VÍO, Marcela (2002). Cambios y enfoques en las pautas de localización industrial en la Región Metropolitana de Buenos Aires. Sus efectos sobre las PYMES. Documento de Trabajo (2). Laboratorio de Investigación sobre Tecnología, Trabajo, Empresa y Competitividad (Littec), Universidad Nacional de General Sarmiento. Disponible en: http://www.littec.ungs.edu.ar/

GARCÍA DELGADO, Daniel (2020). Estado, sociedad y pandemia: ya nada va a ser igual, Facultad Latinoamericana de Ciencias Sociales - FLACSO, Ciudad Autónoma de Buenos Aires, Argentina. Disponible en: http://politicaspublicas.flacso.org.ar/wp-content/ uploads/2020/11/libro-estado-sociedadpandemia.pdf

PONT-VIDAL, Josep (2020). Metrópolis y ciudades en un escenario de incertidumbre. ¿Disponemos de los conceptos necesarios para su observación y análisis? Deliberativa Revista de Estudios Metropolitanos en Gobernanza, 2, e200202-NEM1.T1. Guadalajara, México. Disponible en:https://deliberativa.com/wp-content/uploads/2020/09/DREMeG-e200202-NEM1.T1-Pont.pdf

SUBIRATS, Joan (2015). Nociones conceptuales de gobernanza, política yadministración pública. En Friedrich-Ebert-Stiftung (FES-ILDIS) El rol del Estado: contribuciones al debate. Senplades/FESILDIS, Quito, Ecuador.Disponible en: https://library.fes.de/pdf-files/bueros/quito/12925-20170131.pdf

UIA, Centro de Estudios (2020). "Informe especial. Impacto del Covid en empresas IV. Agosto 2020". Buenos Aires: Unión Industrial Argentina, CEU, agosto, Ciudad Autónoma de Buenos Aires Argentina. Disponible en: https://www.uia.org.ar/centro-de-estudios/3646/informe-de-impacto-del-covid-enempresas-iv/ 


\section{Notas}

${ }^{1}$ Este trabajo resume algunos de los resultados generados en el marco del proyecto "Prevención y monitoreo del COVID-19 en municipios del Conurbano Bonaerense desde una perspectiva multidimensional", financiado por la Agencia Nacional de Promoción Científica y Tecnológica (ANPCyT) de Argentina.

2 El Aislamiento Social, Preventivo y Obligatorio (ASPO) es una medida excepcional que el Gobierno nacional desarrolla para las jurisdicciones con mayor circulación de Covid-19. Dispone que en aquellas zonas del país donde haya transmisión comunitaria del virus (por ejemplo, el AMBA durante los primeros meses de la pandemia), cada persona debe quedarse en su domicilio y que sólo se puede salir para realizar compras básicas, trabajos esenciales o trabajos exceptuados. Por otra parte, el Distanciamiento Social, Preventivo y Obligatorio (DISPO) es una normativa para jurisdicciones donde el riesgo de contagio es más bajo. Plantea que en lugares con sistema de salud adecuado para la demanda sanitaria, que no tengan transmisión comunitaria del virus y en base a otros criterios epidemiológicos, las personas pueden circular, trabajar y realizar sus actividades siempre y cuando guarden siempre dos metros de distancia con otra persona. Del mismo modo, todas las actividades en posibilidades de reabrir deben reorganizarse para garantizar esta norma siendo obligatorio el uso del tapabocas. higiene de manos. ventilación de ambientes y desinfección de superficies (así en lugares cerrados no se pueden reunirse más de 10 personas, cumpliendo el distanciamiento, y nunca superando la ocupación del $50 \%$ de la capacidad del lugar). Esta medida en el AMBA se plasma a partir del mes de noviembre de 2020.

3 Esta área implica el continuo urbano que comienza en la Ciudad Autónoma de Buenos Aires, y se extiende a 24 partidos/municipios de la Provincia de Buenos Aires. Ellos son: Almirante Brown, Avellaneda, Berazategui, Esteban Echeverría, Ezeiza, Florencio Varela, Gral. San Martín, Hurlingham, Ituzaingó, José C. Paz, La Matanza, Lanús, Lomas de Zamora, Malvinas Argentinas. Merlo, Moreno, Morón, Quilmes, San Fernando, San Isidro, San Miguel, Tigre, Tres de Febrero y Vicente López.

${ }^{4}$ El Índice de Producción Industrial Manufacturero (IPI manufacturero) es un indicador de los niveles de actividad de la industria manufacturera para el conjunto de Argentina. 